\title{
A speech analysis-synthesis-editing system based on the ARX speech production model
}

\author{
Weizhong Zhu and Hideki Kasuya \\ Faculty of Engineering, Utsunomiya University, \\ 2753, Ishii-machi, Utsunomiya, 321-8585 Japan
}

(Received 27 May 1997)

\begin{abstract}
We present a new formant-type speech analysis-synthesis-editing system based on the ARX (Auto-Regressive with Exogenous Input) speech production model. One of the key features of the proposed system is implementation of the algorithm that automatically estimates voicing and unvoiced source parameters as well as formant-antiformant parameters of the synthesizer directly from a speech utterance. After automatically estimating the parameters from the utterance, one can manipulate the estimates using a flexible editing tool that has been developed as a part of the system. Using the parameter values so modified, one can synthesize speech with various voice qualities. Since the system has been developed on the MS-Windows platform, it is expected to be a useful tool in various areas of speech research.
\end{abstract}

Keywords : ARX model, Speech analysis, Speech synthesis, Voicing source model, Kalman filter

PACS number: 43. 70. Bk, 43. 70. Jt, 43. 72. Ar

\section{ABBREVIATIONS}

AR filter : auto-regressive filter

ARX model: auto-regressive with exogenous input model

AV : amplitude of voicing source

DEEG : differentiated electroglottograph

EGG : electroglottograph

GCI : glottal closure instant

GOI : glottal open instant

IIR : infinite impulse response

MSEE : mean square equation error

OQ : open quotient

RK model : Rosenberg-Klatt model

RMS : root mean square

T : : pitch period

TL : spectral tilt

\section{INTRODUCTION}

Klatt's speech synthesizer has made a large contribution to various areas of speech research. ${ }^{1)}$ In his synthesizer, however, there are a large number of synthesis parameters to be controlled to generate natural sounding speech. It is not easy to learn to use it effectively. Efforts have been made, on the other hand, to estimate acoustic parameters directly from a natural speech signal and to use the estimated parameters to re-synthesize speech. Imai has proposed a Log Magnitude Approximation (LMA) filter which is used to approximate log magnitude characteristics of a speech signal. ${ }^{2)}$ In the Power Spectrum Envelope (PSE) speech analysis-synthesis system proposed by Nakajima and Suzuki, ${ }^{3)}$ the PSE is modeled by the cosine series which are estimated from a $\log$ power spectrum. All of those methods deal with spectral envelopes which include both voice source and vocal tract characteristics. However, without a voicing source model in the analysis, the voice source and vocal tract characteristics cannot be manipulated independently. Based on the linear prediction covariance analysis method, Kuwabara has shown a method for modifying formant frequencies and bandwidths in a pitchsynchronous analysis-synthesis system. ${ }^{4)}$ "A voice 
source model in the linear prediction analysis, however, is either an impulse train or a white noise source and is thus too simple to represent speech sounds of various voice qualities. In this paper, an ARX (Auto-regressive with exogenous input) speech production model is used to separate voice source characteristics from vocal tract characteristics. ${ }^{5)}$

Formant frequency is one of the most important parameters characterizing phonetic features of speech. Voice source characteristics also significantly contribute to phonetic and voice quality features. An adaptive pitch-synchronous analysis method has been developed ${ }^{5)}$ to estimate the voice source and formant-antiformant parameters separately from a natural speech waveform. In this method, a voicing source waveform is approximated by the Rosenberg-Klatt (RK) model ${ }^{6)}$ and the unvoiced source is represented by a white noise source. To generate a realistic glottal waveform, the RK model includes such parameters as pitch period, glottal open quotient, voicing amplitude and spectral tilt. The Kalman filter algorithm is used to estimate the coefficients of the ARX model and then formant-antiformant parameters are obtained by solving for the roots of the coefficients. This method unfortunately has needed immense computation time. We have devised an improved algorithm which is more compact and much faster than the original one.

In this paper, based on the improved algorithm, we present a new formant-type speech analysissynthesis-editing system. In the system, a flexible editing tool allows the user to manipulate all the estimated acoustic parameters by simply clicking a mouse. One can synthesize speech with various voice qualities by changing the value of the fundamental period, glottal open quotient, spectral tilt parameter, turbulent noise level, and formantantiformant frequencies and bandwidths. A block diagram of the system is illustrated in Fig. 1.

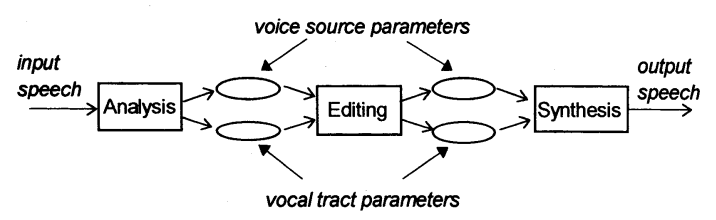

Fig. 1 Block diagram of the analysis-synthesesediting system.
To our knowledge, the analysis-synthesis-editing system including full automatic acoustic analysis to estimate both the voice source and vocal tract parameters has been for the first time developed on the Windows platform. The core of the system is inherited from the previous speech analysis, synthesis and evaluation system. ${ }^{7,8)}$ The system is developed in Borland $\mathrm{C}++$ Object Window and has a user-friendly interface, providing the user with the full advantages of the Windows metaphor.

\section{ARX SPEECH PRODUCTION MODEL}

\subsection{Voicing Source Model}

The RK model is used to represent a differentiated glottal waveform including radiation characteristics, because of its ability to adjust independently both the waveform and spectral slope as well as of its relative ease of implementation. ${ }^{6}$ This model generates a rudimentary waveform defined as

$$
\begin{aligned}
& g(n)= \begin{cases}2 a n-3 b n^{2}, & 0 \leq n \leq T \cdot O Q, \\
0, & T \cdot O Q<n<T,\end{cases} \\
& a=\frac{27 \cdot A V}{4 \cdot\left(O Q^{2} \cdot T\right)}, \\
& b=\frac{27 \cdot A V}{4 \cdot\left(O Q^{3} \cdot T^{2}\right)},
\end{aligned}
$$

where $T$ is a pitch period, $A V$ an amplitude parameter and $O Q$ an open quotient of the glottal open phase to the duration of a complete glottal cycle. The value of $g(n)$ is zero in the closed phase. The differentiated glottal waveform $u(n)$ is generated by smoothing $g(n)$ with a low-pass filter where tilt of the spectral envelope is adjusted by a spectral tilt parameter $T L{ }^{6)}$

\subsection{ARX Model}

Speech production process is modeled as an IIR filter with an equation error as follows,

$$
s(n)+\sum_{i=1}^{p} a_{i} s(n-i)=\sum_{j=0}^{q} b_{j} u(n-j)+e(n),
$$

where $s(n)$ and $u(n)$ denote a speech signal and a differentiated glottal waveform at time $n$, respectively. ${ }^{5)}$ In the equation, $a_{i}$ and $b_{j}$ are filter coefficients, $p$ and $q$ are model orders, and $e(n)$ is an equation error. When $e(n)$ is assumed to be white, the equation is referred to as an ARX model. By performing the $z$-transform on the equation, one gets the following equation,

$$
S(z)=\frac{B(z)}{A(z)} U(z)+\frac{1}{A(z)} E(z),
$$


where $S(z), U(z)$ and $E(z)$ are the $z$-transform of the speech signal $s(n)$, voicing source $u(n)$, and equation error $e(n)$, respectively. $B(z) / A(z)$ indicates the vocal tract transfer function for the voiced sound, whereas $1 / A(z)$ represents the one for the unvoiced sound.

\section{ANALYSIS ALGORITHM}

A speech signal consists of voiced, unvoiced, mixed-voice and silent segments. The analysis frame is synchronized with estimated pitch periods in the voiced and mixed-voice segment, whereas the analysis frame is shifted every $5 \mathrm{~ms}$ in the unvoiced segment. Voiced and mixed-voice segments are specified beforehand by the user with an interactive labeling tool which is implemented as a part of the system. For the voiced speech segment, the key point of the analysis is how to divide the speech signal into the glottal waveform which is approximated by the voicing source parameters of the RK model and the vocal tract characteristic which is represented by the system transfer function $B(z) /$ $A(z)$. An adaptive pitch-synchronous algorithm has been developed to estimate the voicing source and vocal tract parameters directly from the speech signal. ${ }^{5)}$ Figure 2 shows the block diagram of the analysis procedure for a voiced segment. The voicing source parameters in the current analysis period are optimized by minimizing the mean square equation error (MSEE). In order to estimate the IIR filter coefficients, the filter is expanded into a timevariant system so that a Kalman filter algorithm can be used. In the algorithm, an input signal is a differentiated glottal waveform $u(n)$ approximated by optimized values of the RK model parameters and the output is an observed speech signal $s(n)$.

In the estimation of the RK parameters, the previous optimization method ${ }^{5)}$ has employed, on the basis of the MSEE criterion, the simulated anneal-

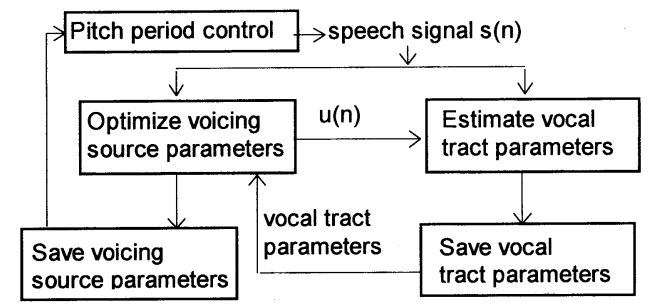

Fig. 2 Block diagram of the analysis procedure for a voiced speech segment. ing algorithm which in general takes much computation time. Taking sensitivity of each of the RK parameters to the MSEE value into account, we present an improved algorithm where only $O Q$ is optimized and the other parameters are estimated in different ways to be described below.

\subsection{Estimation of Voicing Source Parameters}

Optimization of the RK voicing source parameters is performed so as to minimize the MSEE value as mentioned above. The MSEE is a function of the RK parameters, $A V, T L$ and $O Q . \quad A V$ is estimated as the coefficient $b_{0}$ of $B(z)$ using the Kalman filter algorithm. $T L$, on the other hand, is estimated from the inverse filtered signal using the method proposed by Lu et al. ${ }^{9)}$ These simplifications conserve computation time significantly without sacrificed accuracy. $O Q$, which is important and very sensitive to the MSEE, is estimated by an optimization method. The estimation is performed following six steps bellow on a cycle-to-cycle basis (see Fig. 3).

(1)Take an analysis window length identical to the previous pitch period $T(n-1)$ with a starting point of $G O I(n)$ (glottal open instant).

(2)Inverse-filter the speech signal in the current analysis window by using the vocal tract parameters in the previous period and estimate $T L$ from the inverse filtered signal. ${ }^{9)}$

(3)Generate candidates of the RK waveform $u(n)$ by varying $O Q$ in a certain range, with a fixed value of $A V$ and $T L$ estimated in step (2) and calculate MSEE's for all the candidates. Find $O Q_{\text {MSEE }}$ that has the minimum MSEE value and define $G C I(n)$ (glottal closure instant) as follows,

$$
G C I(n)=G O I(n)+T(n-1) \cdot O Q_{\mathrm{MSEE}} .
$$

(4)Define the pitch period $T(n)$ as the interval

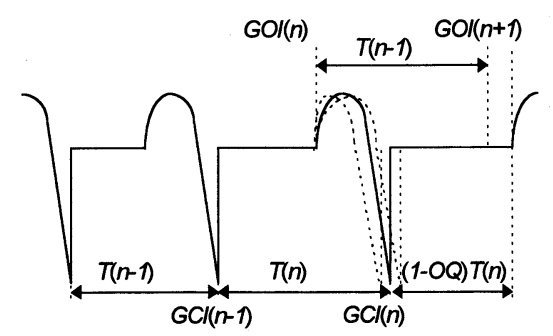

Fig. 3 Estimation of glottal closure instant $(G C I)$ and pitch period. 
between two successive $G C I$ 's,

$$
T(n)=G C I(n)-G C I(n-1) .
$$

(5)Further optimize $G O I(n)$ by fixing $A V, T L$ and $G C I(n)$ obtained in step (3), using the same procedure as in step (3).

(6)Determine the starting point for the next analysis period $G O I(n+1)$ as

$$
G O I(n+1)=G C I(n)+(1-O Q) \cdot T(n) .
$$

In addition to the glottal waveform, a glottal noise component defined by the noise amplitude parameter $(N A)$ is estimated from the prediction error in the glottal open phase as follows,

$$
N A=\sqrt{\frac{1}{N} \sum_{i=0}^{N-1} e^{2}(n)},
$$

where $N$ is the data length within the open interval and $e(n)$ is the prediction error.

\subsection{Estimation of Vocal Tract Parameters}

In order to estimate the coefficients of the vocal tract transfer function, used is the same algorithm as the one proposed by Ding et al. ${ }^{5)}$ except that the system gain parameter $b_{0}(n)$, being related to the amplitude of the glottal waveform, is newly introduced in the equation. The time-varying coefficient vector of the transfer function $X(n)$ and data vector $C(n)$ are respectively defined as

$$
\begin{gathered}
X(n)=\left\{a_{1}(n), \ldots, a_{p}(n), b_{0}(n), \ldots, b_{q}(n)\right\}^{\mathrm{T}}, \quad(8) \\
C(n)=\{-s(n-1), \ldots,-s(n-p), u(n), \ldots, u(n-q)\}^{\mathrm{T}} .
\end{gathered}
$$

The Kalman gain and correlation matrix are defined as $G(n)$ and $K(n)$, respectively. The Kalman filtering algorithm ${ }^{5)}$ for one pitch cycle $T$ is implemented using the following equations,

$$
\begin{aligned}
& G(n)=K(n-1) C(n)\left[C^{\mathrm{T}}(n) K(n-1) C(n)+1\right]^{-1}, \\
& e(n)=s(n)-C^{\mathrm{T}}(n) X(n) \\
& X(n+1)=X(n)+G(n) e(n) \\
& K(n)=K(n-1)-G(n) C^{\mathrm{T}}(n) K(n-1),
\end{aligned}
$$

where $n=1,2, \ldots, T$.

Before proceeding to the next period, the correlation matrix is further updated point by point with the following equation,

$$
K(n+1)=K(n)+a \cdot \operatorname{diag}(K(n)), \quad \alpha=0.01 .
$$

Initial values of the correlation matrix are

$$
K(0)=\operatorname{diag}(100,100, \ldots, 100) .
$$

Although the time-varying filter coefficients are computed at every point in the iteration as shown in Eq. (10), the formant-antiformant values are computed by solving for the roots of the polynomials at only three points within the pitch period, i.e. the last of the period, the middle of the open phase and the middle of the closed phase. In the theory, a set of formant values estimated at the last analysis point should be the best candidate but in practice it fails once in a long while. Therefore, a set of formant-antiformant values which has the maximum number of formants is saved as the representative of the vocal tract parameters within the period. This is because (1) calculation of formant values at every point takes immense time and a large capacity for the storage of the parameters is needed, (2) an estimated vocal tract filter is not always stable around the glottal closure instant, and finally (3) it is not unreasonable to assume time-invariant nature of the vocal tract movement within one pitch period. From preliminary experiments, we have also found that the filters estimated at the three points within the period mentioned above are stable. ${ }^{10)}$

\subsection{Selection of Model Order}

For the selection of the optimum order of the model, the method by Ding et al. is used. ${ }^{5)}$ If it does not work well, then the user can easily change the order in the dialogue box in the system.

\section{SYNTHESIS STRATEGY}

A cascade formant synthesizer is used to synthesize the voiced and unvoiced speech. This simplicity is the advantage of our system over the one by Klatt $^{1)}$ in which a complicated cascade and parallel structure is implemented. The RK model is used to synthesize the voiced speech, whereas the Msequence white noise is used to synthesize the unvoiced speech.

\subsection{Cascade Formant Synthesizer}

The synthesizer is composed of the second-order resonators in cascade form. The system function of each resonator is expressed as 


$$
\begin{aligned}
& H(z)=\frac{a}{1-b z^{-1}-c z^{-2}}, \\
& b=2 \exp \left(-\pi B / f_{\mathrm{s}}\right) \cos \left(2 \pi F / f_{\mathrm{s}}\right), \\
& c=-\exp \left(-2 \pi B / f_{\mathrm{s}}\right), \\
& a=1-b-c,
\end{aligned}
$$

and that of anti-resonator as

$$
\begin{aligned}
& H(z)=a^{\prime}+b^{\prime} z^{-1}+c^{\prime} z^{-2}, \\
& a^{\prime}=1 / a, b^{\prime}=-b / a, c^{\prime}=-c / a,
\end{aligned}
$$

where $F, B$ and $f_{\mathrm{s}}$ are formant frequency, bandwidth and sampling frequency, respectively.

\subsection{Adjustment of Voice Amplitude Parameter}

In the formant synthesizer, gain of each secondorder resonator at $0 \mathrm{~Hz}$ is always $0 \mathrm{~dB}$. But the amplitude of the vocal tract transfer function $B(z) /$ $A(z)$ estimated by the Kalman filter at $0 \mathrm{~Hz}$ may not always be equal to $0 \mathrm{~dB}$. As a result, an estimated amplitude parameter $A V,\left(=k b_{0}, k\right.$ : constant), can not be used directly in such a formant synthesizer. There are three ways to adjust this $A V$ parameter: (1) minimize difference between the root mean square (RMS) values for the original and synthesized speech, (2) minimize the mean square error between the original and synthesized speech, and (3) minimize difference of positive or negative peak values between the original and synthesized speech. The RMS criterion is used in our method since we have found that it yields reasonable envelops of $A V$ sequences as compared with the others.

\subsection{Synthesis with Glottal Closure Instants}

Since we define the pitch period $T$ as an interval between the two successive GCI's in the synthesis, the RK glottal waveform of one pitch period begins with the close phase of the previous cycle, followed by the RK waveform of the open phase of the current cycle (see Fig. 4). In this way, GCI's keep

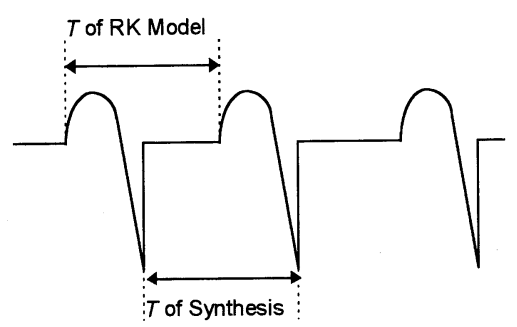

Fig. 4 Procedure for synthesizing speech with GCI's. the same values, even when values of $O Q$ are changed.

\section{EDITING TOOLS}

Each of the estimated voice source and formantantiformant parameters is re-sampled every $5 \mathrm{~ms}$ and displayed on the graphical window. An example of the trajectories of the formant parameters estimated from a Japanese sentence /aoiueoie/ ("Say blue top" in English) is shown in Fig. 5. An editing tool can be applied to manipulate the parameters on the graphical window.

\subsection{Editing Parameters}

A number of functions have been developed to edit the parameters being displayed as the points on the graphical window. ${ }^{7,8)}$ The following is the list of the functions.

1. Modify the value: Move the mouse to the point to be modified, press the left button and drag the mouse to a new value.

2. Add a new value: Press the Shift key and click the left button at the point of a new value.

3. Delete the value: Move the mouse to the point to be deleted, press the Ctrl key and click the left button.

4. Modify the multiple values: Double-click at the start point and then at the end point. The points between the start and the end are linearly' interpolated.

5. Scale the values: Double-click at the first point and then at the last point on the window and input a scaling factor.

The right button is used to modify the formant

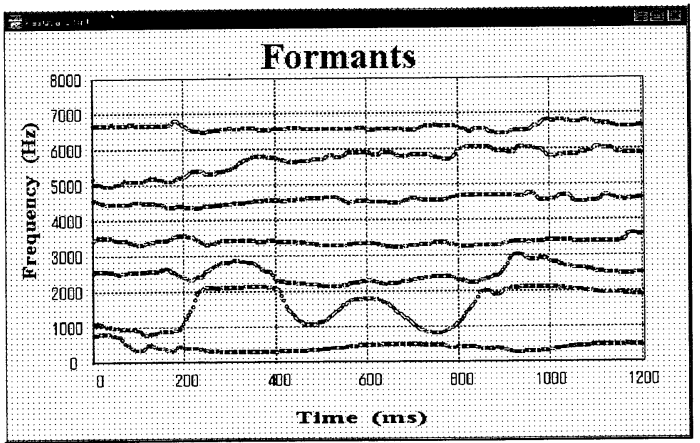

Fig. 5 Trajectories of the formant parameters estimated from a Japanese sentence/aoiueoie/ ("Say blue top" in English). 
bandwidth on the formant window.

\subsection{Saving, Printing and Linking to Other Appli- cations}

A graphical window can be saved as a text file which contains the main and the $x-y$ tittles as well as the parameter values. A user can restore a graphical window from the saved text file. The graphical window can be easily merged into a word processor, such as MS-WORD, while all the data files can also be accessed by other application programs, such as MS-EXCEL, to do further analysis.

\subsection{Other Useful Tools}

The system is also equipped with many basic speech analysis, waveform editing and labeling, and filter design tools. ${ }^{7,8)} \quad$ Using the basic speech analysis tool, one can get the FFT/LPC spectrum and sound spectrogram by simply clicking a mouse. Any two of the FFT and LPC spectra can be superimposed on the same graphical window. With the powerful editing tools, one can copy, cut, and paste between the different speech windows and modify the amplitude of a speech waveform on the point-bypoint basis. By clicking one of the low-pass, bandpass and high-pass menus and entering specified data, one can design a filter and apply it to a speech signal immediately. The system also provides functions for replaying and recording speech signals through the sound board incorporated in the Windows system.

\section{EVALUATION}

Since we use the same algorithm as the one proposed by Ding et $a l{ }^{5)}$ in the estimation of the vocal tract parameters, required is evaluation of the new algorithm used to estimate the voice source parameters. We also give an example of analysissynthesis-editing of a speech signal to show the state of the art of the system.

\subsection{Voice Source Parameters Estimation}

A sentence /aoiue/ ("blue top" in Japanese) uttered by a male was used to compare the $G C I$ estimates between the proposed algorithm and the previous one. A two-channel signal (speech and electroglottograph (EGG) signal) was recorded in a soundproof room and was sampled at a sampling frequency of $14.7 \mathrm{kHz}$. By using the negative peaks of differentiated EGG (DEGG) as the standard
GCI's, the results of the two algorithms are shown in Fig. 6, where the histogram shows counts of the differences between the standard and estimated $G C I$ 's in points. It is not unfair to say that both algorithms estimate the GCI's accurately. But the new algorithm is about 10 times faster than the previous one.

$O Q$ and $T L$ were evaluated using a synthesized speech signal. These are estimates since it is very difficult to specify the true values of both $O Q$ and $T L$ from the EGG signal. A synthesized speech signal of Japanese vowel /a/ was generated by using varying patterns of $O Q$ and $T L$ while keeping other parameters constant. The estimates of $O Q$ and $T L$ resampled in $5 \mathrm{~ms}$ are shown in Fig. 7 and Fig. 8, respectively together with their test patterns, where

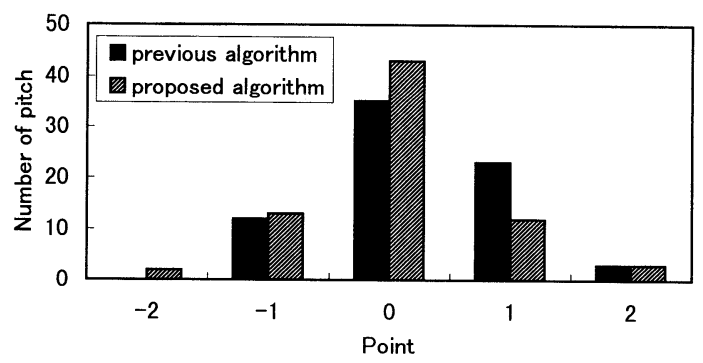

Fig. 6 Comparison of $G C I$ estimations between the previous and the proposed algorithms.

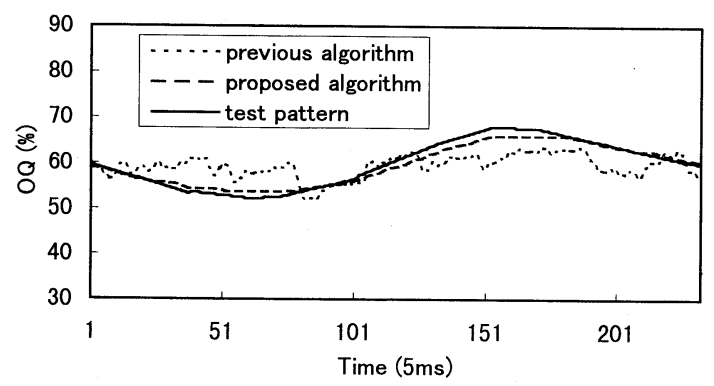

Fig. 7 Estimates of $O Q$ and test pattern.

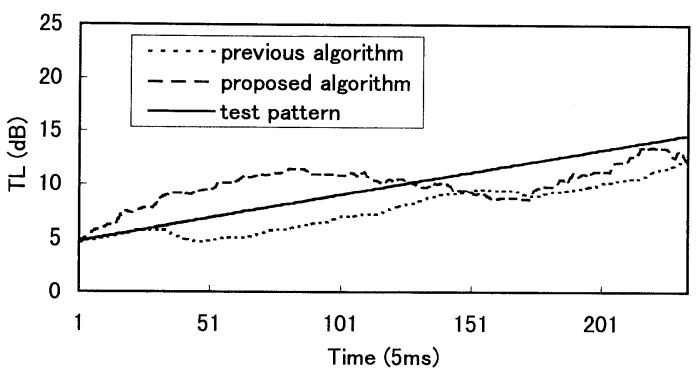

Fig. 8 Estimates of $T L$ and test pattern. 


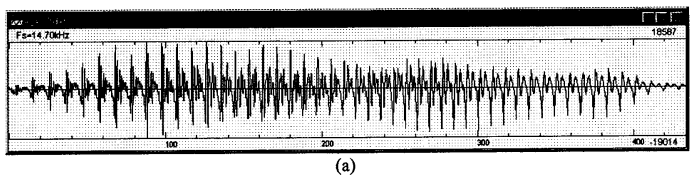

(a)
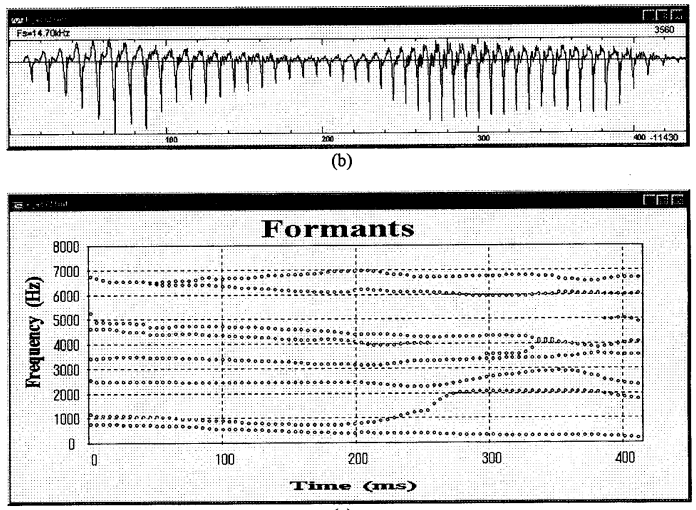

(c)

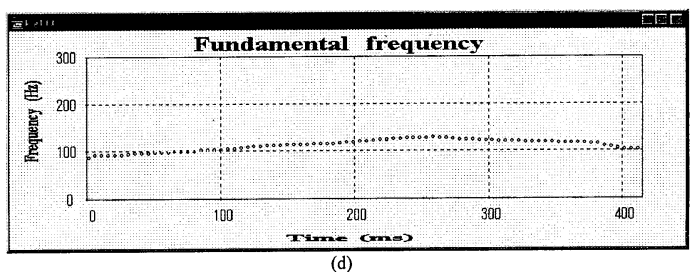

(d)

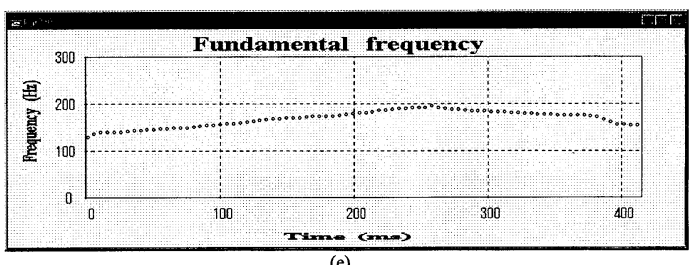

(e)

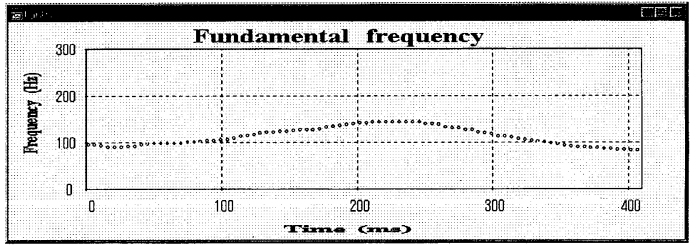

(f)

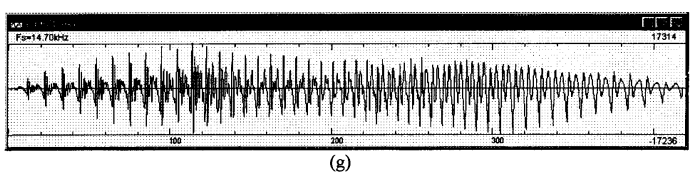

Fig. 9 (a) Speech waveform of /aoi/ with no accent. (b) Estimated differentiated glottal waveform. (c) Estimated formant trajectories. (d) Estimated $F_{0}$ contour. (e) $F_{0}$ contour scaled up by a factor of 1.5 . (f) $F_{0}$ contour modified to place the accent on the second syllable. (g) Synthesized speech waveform with modified $F_{0}$ contour. the values estimated by previous algorithm are drawn as a dotted line, those of proposed algorithm as a dashed line and the test values as a solid line. It is shown that the $O Q$ estimated by the proposed algorithm tracks well the change of the test $O Q$. The overall trend of the $T L$, estimated by the proposed algorithm reflects the test $T L$, but it seems to be influenced by the variation of $O Q$ and the accuracy seems to be similar between the new and previous algorithms. As a conclusion, without sacrificed accuracy, we have introduced a new algorithm to estimate voicing source parameter which is more compact and much faster than the original one.

6.2 Example of Analysis-Synthesis-Editing of a Speech Signal

Some Japanese words have different meanings when the accent is on the different syllable, even if they have same phonemic structure. Generally, the phonemic structure of a word is determined by vocal tract characteristics (formant values), while the accent is associated with voice source characteristics (fundamental frequency $\left(F_{0}\right)$ ). With or without the accent on the second syllable, a word /aoi/ may have a meaning of blue or hollyhock in English. An experiment was made on the analysis of speech "hollyhock" and synthesis of speech "blue" by modifying the $F_{0}$ values. A speech waveform of an utterance of /aoi/ (hollyhock) spoken by a male talker is shown in Fig. 9 (a). Based on the ARX analysis, we obtained the differentiated glottal waveform, formant trajectories and $F_{0}$ contour, which are shown in Fig. 9 (b), (c) and (d), respectively. The $F_{0}$ contour scaled up by a factor of 1.5 , which is shown in Fig. 9 (e), was obtained by simply double-clicking at the first and then at the last point on the $F_{0}$ window and entering a scaling factor of 1.5. Using the editing tools mentioned in section 5.1, we modified the values of $F_{0}$ to the pattern with the accent on the second syllable (Fig. 9 (f)). A natural sounding synthesized speech "blue" (Fig. 9 (g)) was generated from the modified $F_{0}$ contour. Similarly, the modification can be applied to the formant trajectories as well as the other parameters.

\section{SUMMARY}

A new speech analysis-synthesis-editing system based on the ARX speech production model has been developed on the MS-Windows platform. 
One of the advantages of this system is that it separates the voice source characteristics from those of the vocal tract. Resynthesis of speech can be made after independent manipulation of the voice source and vocal tract parameters, to produce speech with various voice qualities. It is expected to be a useful tool in various areas of speech research.

\section{ACKNOWLEDGMENTS}

The authors would like to thank Dr. W. Ding and Mr. T. Matsushita for providing Kalman filtering and formant synthesis programs. Thanks are also due to Mr. L. Mutenda who gave valuable comments to improve the paper.

\section{REFERENCES}

1) D. H. Klatt, "Software for a cascade/parallel formant synthesizer," J. Acoust. Soc. Am. 67, 971-995 (1980).

2) S. Imai, "Log magnitude approximation (LMA) filter," IEICE J63-A, 886-893 (1980) (in Japanese).

3) T. Nakajima and T. Suzuki, "Power spectrum envelope (PSE) speech analysis-synthesis system," J. Acoust. Soc. Jpn. (J) 44. 824-832 (1988) (in Japanese).

4) H. Kuwabara, "A pitch-synchronous analysis/synthesis system to independently modify formant frequencies and bandwidths for voiced speech," Speech Commun. 3, 211-220 (1984).

5) W. Ding, H. Kasuya, and S. Adachi, "Simultaneous estimation of vocal tract and voice source parameters based on an ARX model," IEICE Trans. Inf. Syst. E78-D, 738-743 (1995).

6) D. H. Klatt and L.C. Klatt, "Analysis, synthesis, and perception of voice quality variations among female and male talkers," J. Acoust. Soc. Am. 87, 820-857 (1990).

7) W. Zhu, Y. Kikuchi, Y. Endo, H. Kasuya, M. Hirano, and M. Ohashi, "An integrated acoustic evaluation system of pathologic voice," ICSLP-94, Yokohama, 1983-1986 (1994).

8) W. Zhu and H. Kasuya," A new speech synthesis system based on the ARX speech production model," ICSLP-96, Philadelphia, 1413-1416 (1996).
9) J-L. Lu, Y. Ando, and H. Kasuya, "Study of voicing source characteristics based on a semiautomatic glottal inverse filtering method," J. Acoust. Soc. Jpn. (J) 48, 642-648 (1992) (in Japanese).

10) M. Matsuda, T. Matsushita, W. Ding, and H. Kasuya, "Examination of dynamic characteristic of vocal tract and voice source parameters by time variant ARX analysis," Proc. Spring Meet. Acoust. Soc. Jpn., 293294 (1996) (in Japanese).

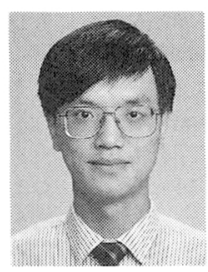

Weizhong Zhu received the B. S. in Computer Science from Fudan University, China, in 1982. He joined the Shanghai Institute of Computing Technology (SICT), China, as research assistant in 1982, where he was engaged in research and development of computer application systems. From 1989 to 1993 , he has been a associate researcher at same institute. He is currently a candidate of Ph. D. in Production and Information Sciences at Utsunomiya University, Japan. His current research interests include speech analysis-synthesis, speech coding, voice quality conversion and software tools for speech research. He is a member of the Acoustical Society of Japan.

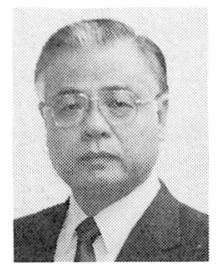

Hideki Kasuya received the B.S., M. S., and Ph. D. degrees in Electrical Communication Engineering all from Tohoku University, Sendai, Japan, in 1963, 1965, and 1970, respectively. In 1968 he joined as Research Associate the Research Institute of Electrical Communication at Tohoku University, where he was primarily engaged in speech analysis, perception and recognition. From 1974 to 1977 he was Visiting Researcher at the Speech Communications Research Laboratory, Inc., California, U. S. A., working on speech recognition. Since 1978 he has been with the Faculty of Engineering, Utsunomiya University, where he is now a professor in the Department of Electrical and Electronic Engineering. His research interests include various areas of speech science and technology, digital signal processing, and image processing. 